\title{
Medical-Surgical Nursing
}

National Cancer Institute

\section{Source}

National Cancer Institute. Medical-Surgical Nursing. NCI Thesaurus. Code C20874.

Medical-Surgical Nursing includes the provision of care to individuals who have a known or predicted physiological alteration. Working within an interdisciplinary team and guided by theory and research, medical-surgical nursing practice considers all influences on health status and the related social and behavioral problems arising because of the patient's physiological condition. Medical-surgical nursing is practiced in primary, acute, or long-term nursing care facilities. (from ANCC) 